\title{
A computational study on the excited state properties of a cationic cyanine dye: TTBC
}

\author{
Sila Karaca, Nuran Elmac1* \\ Department of Chemistry, Izmir Institute of Technology, Gülbahçe-Urla-35430 Izmir, Turkey
}

\section{A R T I C L E I N F O}

\section{Article history:}

Received 13 September 2010

Received in revised form 13 December 2010

Accepted 20 December 2010

Available online 30 December 2010

\section{Keywords:}

TDDFT

DFT

Excited state

Cyanine dye

TTBC

JC-1

\begin{abstract}
A B S T R A C T
The ground and excited state behaviors of a biologically important mitochondria selective dye, 5,5',6,6'tetrachloro-1, $1^{\prime}, 3,3^{\prime}$-tetraethyl-benzimidazolo carbocyanine (TTBC), were investigated in order to give useful information related to the enhancement of its electronic and optical properties. DFT and TDDFT levels of theory with different functionals and basis sets were used. The results obtained by using PBEPBE/6-31+G(d,p)/C-PCM are in good agreement with the experiments. The potential energy surface sections of ТТВC were explored and the geometry optimized in the first excited state, $S_{1}$, to analyze the fluorescence spectrum of the dye. There are two distinct minima on the $S_{1}$ surface, one located near the ground state geometry and an energetically favorable twisted geometry, having a charge transfer character. The observation of similar ground and excited state dipoles as well as solvent-independent fluorescence and absorption wavelengths reveals that there is no competition between the two minima; the emission is dominantly coming from the more planar configuration.
\end{abstract}

(c) 2010 Elsevier B.V. All rights reserved.

\section{Introduction}

Cyanine dyes are synthetic dyes with the general formula, $\mathrm{R}_{2} \mathrm{~N}[\mathrm{CH}=\mathrm{CH}]_{n} \mathrm{CH}=\mathrm{N}^{+} \mathrm{R}_{2}$. They consist of two nitrogen centers bonded to each other by a conjugated chain of the odd number of carbon atoms with one of the centers being positively charged $[1,2]$. The first cyanine was synthesized in the 1800s [3]. They are widely used as sensitizers for photographic films [4], laser dyes [5,6], anti-tumor agents [7], non-linear optics [8], optical disk recording media [9] and as fluorescence probes for biological molecules [10]. They are ideal fluorescent reagents, because of their high extinction coefficient and good quantum yield. In addition, they exhibit absorbance and emission wavelength maxima in the range of $400 \mathrm{~nm}$ to $900 \mathrm{~nm}$ [11].

A well known cyanine dye, which has a delocalized positive charge is $1,1^{\prime}, 3,3^{\prime}$-tetraethyl-5,5',6,6'-tetrachlorobenzimidazolocarbocyanine (TTBC or JC-1). Its chemical structure is shown in Fig. 1. It is used as a molecular probe especially for monitoring mitochondrial membrane potential $(\Delta \Psi)$. A high $\Delta \Psi$ indicates that the dye is in the J-aggregate [12,13] form, emitting at $590 \mathrm{~nm}$ and that the cell is alive, whereas a low $\Delta \Psi$ is a sign of apoptosis and TTBC exists in the monomer form, emitting at $527 \mathrm{~nm}$ [14-16]. The advantage of TTBC rather than the other

\footnotetext{
* Corresponding author. Tel.: +90 232750 7532; fax: +90 2327507509 .

E-mail address: nuranelmaci@iyte.edu.tr (N. Elmacı).
}

carbocyanines is that the status of mitochondria can be monitored with both low and high membrane potentials [17].

The purpose of this study is to investigate the ground and excited state behaviors of the well known mitochondria selective cyanine dye, TTBC by the use of quantum chemical methods. The simulated absorption and fluorescence spectrum of TTBC will be compared with the experimental spectra reported by Özçelik [18]. This versatile organic dye is an important biological molecular probe; it is envisioned that the knowledge gained from our work here may benefit the use and development of the optical and spectroscopic properties of this and similar dyes. The theoretical investigation of the ground and excited state molecular and electronic structures of the TTBC dyes would shed light on what may be required to enhance their functions and applications.

Time-Dependent Density Functional Theory [19-21] (TDDFT) has been widely used for the excited state energies of organic molecules recently [22-30]. It is a powerful tool with low computational cost. Jacquemin, Preat, Perpéte and Adamo have published a series of works on various organic dyes concerning the weakness and strength of TDDFT as well as the efficiency, and reliability of DFT functionals for spectroscopic calculations [31-40]. The calculated transition energies of many organic dyes including cyanines with TDDFT are in good agreement with experiment although some limitations of TDDFT such as extended $\pi$ systems $[41,42]$, Rydberg states [43,44], double excitations [45-47], and charge transfer [48-50] are reported. 


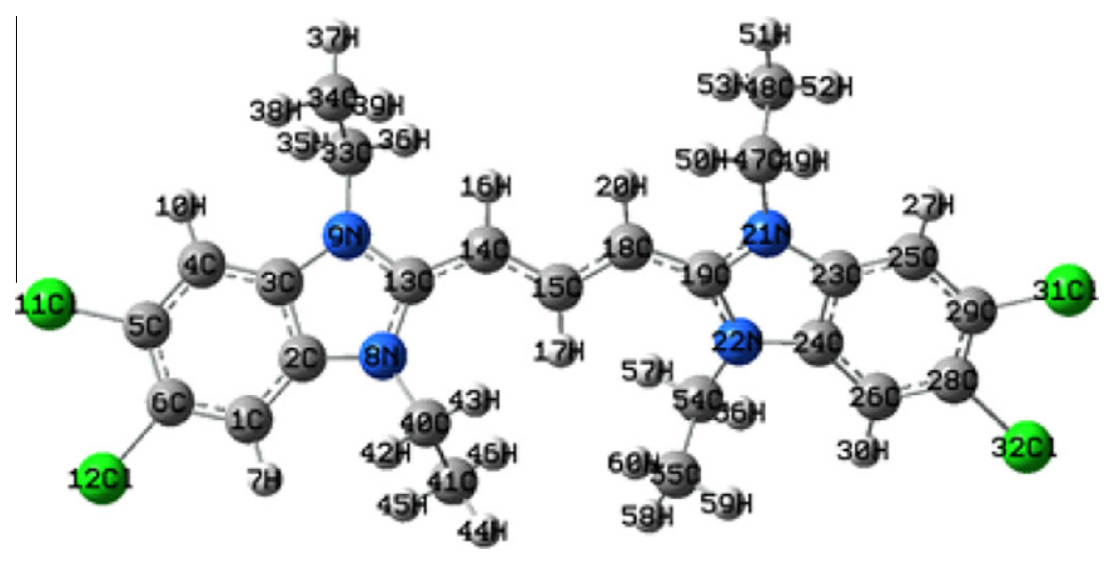

Fig. 1. Cationic structure of TTBC.

\section{Computational details}

All the computations have been carried out with the Gaussian09 program suite [51]. We have performed computations for the geometry optimizations and potential energy surface sections of TTBC in both gas phase and solvent at the ground and excited states. The frequency calculations have also been done to check imaginary eigenvalues for the confirmation of minimum structure. The iodide ion (counter ion) of the TTBC has been ignored in all calculations. Semi-empirical methods AM1 and PM3, ab initio method HF (Hartree-Fock), and DFT (Density Functional Theory) have been chosen in the optimization part with different functionals and basis sets. The DFT functionals used in the calculations are the gradient-corrected exchange-correlation functional of Perdew, Burke and Ernzerhof, PBEPBE [52], and hybrid functionals (25\% exchange and $75 \%$ correlation). PBE1PBE [53,54] and Becke Three Parameter Lee, Yang, and Parr, B3LYP [55], The Coulomb-attenuated hybrid exchange-correlation functional, CAM-B3LYP [56]. Time-Dependent Density Functional Theory (TDDFT) has been used for the excited state calculations. The solvation effects have been investigated using the Integral Equation Formalism Polarizable Continuum Model [57-60] (IEF-PCM) and Conductor-like Polarizable Continuum Model $[61,62]$ (C-PCM).

\section{Results and discussion}

\subsection{Ground state calculations}

Various levels of theory have been used for the ground state, $S_{0}$, structure determination. The calculated geometrical parameters have been compared with X-ray data [63] by performing linear regression analysis without hydrogen atoms (Table 1 ). The orientation of the ethyl groups on the calculated TTBC geometry are different from the X-ray data may be reflected as a decrease in the

Table 1

Correlation coefficients of bond distances and bond angles for various methods used in geometry optimization of TTBC.

\begin{tabular}{lll}
\hline Methods & $R^{2}$ with bond distances & $R^{2}$ with bond angles \\
\hline AM1 & 0.9395 & 0.9505 \\
PM3 & 0.9265 & 0.9756 \\
STO-3G & 0.9822 & 0.9852 \\
HF/6-31G** & 0.9853 & 0.9794 \\
B3LYP/6-31G & 0.9894 & 0.9813 \\
CAM-B3LYP/6-31G & 0.9887 & 0.9831 \\
PBEPBE/6-31G** & 0.9858 & 0.9822 \\
PBE1PBE/6-31G** & 0.9856 & 0.9832 \\
\hline
\end{tabular}

correlation coefficients. The geometry optimizations were performed starting with different orientations of alkyl groups but it has been observed that the direction of ethyl groups does not affect the stability, but rather have free rotation. When the bond distances were used in the regression analysis, the three largest correlation coefficients, $R^{2}$, have been found for the calculations at B3LYP, CAM-B3LYP, and PBEPBE level of theory. However, the order becomes HF/STO-3G, PBE1PBE, and CAM-B3LYP according to the bond angles. The PM3 result is in best agreement with the X-ray dihedral angle for the dihedral angle $\left(\theta_{1}=\right.$ N8-C13-C14-15), which shows deviation from planarity as shown in Table 2 . On the other hand, DFT values for this torsional angle differ from the experimental value by about $15^{\circ}$, which might be explained by the packing effect in crystals. It should be noted that the geometry optimization results obtained for TTBC with the B3LYP/6-31G(d,p) method have been reported in our previous work, which is related to the substituent effects on the UV-Vis absorption wavelength of that dye [64].

These findings state that any of the given DFT methods with the given functionals can be used to describe the TTBC ground state properties since there is no significant difference in the correlation coefficients.

\subsection{Absorption calculations}

We have used both TDDFT and CIS methods to investigate the excited state behavior of TTBC; however, the $\lambda_{\max }(330 \mathrm{~nm})$ value obtained by CIS is much lower than experimental results (514$522 \mathrm{~nm}$ ). We concluded that the CIS method is not reliable for the excited state calculations of TTBC.

The effects of split valence, polarized basis sets, diffuse functions, and DFT functionals on the excited state calculations of TTBC have been examined to propose a valid computational level. The B3LYP/6-31G $(d, p)$ ground state equilibrium structure is used in these calculations. The $\lambda_{\max }$ values and corresponding oscillator strengths obtained by these calculations are listed in Table 3. All the oscillator strengths are about two arbitrary units.

The B3LYP functional has been used with different basis sets in the excited state calculations. The calculated $\lambda_{\max }$ increased by $6 \mathrm{~nm}$ when diffusion functions were added to the heavy atoms. Use of the triple-split valence basis set (6-311) red-shifted $\lambda_{\max }$ by $5 \mathrm{~nm}$ when compared to the double-split basis set, (6-31) where there is no diffusion function. However, when a diffusion function is used with the double-split basis set, there is only a 1-2 nm difference in $\lambda_{\max }$ values. Both diffusion- and split-valence effects redshifted $\lambda_{\max }$ by $8 \mathrm{~nm}$ (Table 3 ). Addition of polarization effects on the heavy atoms modified $\lambda_{\max }$ by $4 \mathrm{~nm}$. In contrast there is almost 
Table 2

Dihedral angle of TTBC obtained by different methods.

\begin{tabular}{|c|c|c|c|c|c|c|c|c|c|}
\hline Dihedral angle $\left(\theta_{1}\right)$ & AM1 & PM3 & HF/STO-3G & $\mathrm{HF} / 6-31 \mathrm{G}^{* *}$ & B3LYP/6-31G** & CAM-B3LYP/6-31G ${ }^{* *}$ & PBEPBE/6-31G ${ }^{* *}$ & PBE1PBE/6-31G ${ }^{* *}$ & $X$-ray[63] \\
\hline $\mathrm{N} 8-\mathrm{C} 13-\mathrm{C} 14-\mathrm{C} 15$ & 25 & 6 & 13 & 25 & 20 & 21 & 19 & 20 & 4 \\
\hline
\end{tabular}

Table 3

Diffuse function, split valence, polarization and DFT functional effects on $\lambda_{\max }$ (in nm) and oscillator strengths $(f)$ of TTBC.

\begin{tabular}{llll}
\hline & & $\lambda_{\max }$ & $f$ \\
\hline Diffuse functions \& & B3LYP /6-31G(d,p) & 433 & 1.8928 \\
split valences & B3LYP /6-31+G(d,p) & 439 & 1.8670 \\
& B3LYP /6-31++G(d,p) & 440 & 1.8644 \\
& B3LYP /6-311G(d,p) & 438 & 1.8857 \\
& B3LYP /6-311+G(d,p) & 441 & 1.8641 \\
& B3LYP /6-311++G(d,p) & 441 & 1.8626 \\
Polarization functions & B3LYP/6-31G & 428 & 1.9286 \\
& B3LYP/6-31G(d) & 432 & 1.9015 \\
& B3LYP/6-31G(d,p) & 433 & 1.8928 \\
& B3LYP/6-31G(2d,2p) & 435 & 1.8973 \\
& B3LYP/6-31G(df,pd) & 433 & 1.8881 \\
& B3LYP/6-31G(2df,2pd) & 435 & 1.8900 \\
& B3LYP/6-31G(3d,3p) & 439 & 1.8801 \\
& CAM-B3LYP/6-31G(d,p) & 409 & 1.9341 \\
& MPW1PW91/6-31G(d,p) & 424 & 1.9336 \\
& PBE1PBE/6-31G(d,p) & 424 & 1.9346 \\
& B3PW91/6-31G(d,p) & 432 & 1.9053 \\
& B3LYP/6-31G(d,p) & 433 & 1.8928 \\
& HCTH/6-31G(d,p) & 466 & 1.7441 \\
& PBEPBE/6-31G(d,p) & 470 & 1.7379 \\
\hline
\end{tabular}

no effect of the polarization and diffusion functions on the hydrogen atoms. It has been observed that the largest change is found around $11 \mathrm{~nm}$ when the basis set is extended with higher-order polarizations (2d,2p; df,pd; 2df,2pd; 3d,dp); however, to make computational times more reasonable, the $6-31 \mathrm{G}(\mathrm{d}, \mathrm{p})$ basis set was chosen for our calculations.

After choosing the basis set, the DFT functional effect was studied by changing exchange and hybrid functionals in excited state computations. According to Table 3, the change of the functionals significantly affected the $\lambda_{\max }$ much more than the diffusion and polarization extensions without additional computational demand. The highest wavelength $(470 \mathrm{~nm})$ was obtained with PBEPBE functional. It differs by about $40 \mathrm{~nm}$ from B3LYP result.

To potentially improve on this, different functionals for both the ground and the excited state computations were employed after considering the observation of the functional effect on the excited state. Table 4 shows the $\lambda_{\max }$ values calculated with various functionals in the ground state structure optimization and excited state calculations. When the PBEPBE functional was used in both the ground and excited state, $\lambda_{\max }$ was found to be $477 \mathrm{~nm}$. Although the $\lambda_{\max }$ value remained almost the same by the extension of the basis set with diffuse functions in the ground state optimization,

Table 4

Effect of DFT functionals used in the geometry optimization in $S_{0}$ and absorption $\lambda_{\max }$ (in $\mathrm{nm}$ ) and oscillator strengths $(f)$ calculations of TTBC.

\begin{tabular}{llll}
\hline Ground state & Excited state & $\lambda_{\max }$ & $f$ \\
\hline B3LYP/6-31G(d,p) & B3LYP/6-31G(d,p) & 433 & 1.8928 \\
PBE1PBE/6-31G(d,p) & B3LYP/6-31G(d,p) & 430 & 1.8840 \\
PBEPBE/6-31G(d,p) & B3LYP/6-31G(d,p) & 440 & 1.8862 \\
B3LYP/6-31G(d,p) & PBEPBE/6-31G(d,p) & 470 & 1.7379 \\
PBEPBE/6-31G(d,p) & PBEPBE/6-31G(d,p) & 477 & 1.7320 \\
PBEPBE/6-31+G(d,p) & PBEPBE/6-31G(d,p) & 478 & 1.7330 \\
PBEPBE/6-31G(d,p) & PBEPBE/6-31+G(d,p) & 485 & 1.7044 \\
PBEPBE/6-31+G(d,p) & PBEPBE/6-31+G(d,p) & 486 & 1.7042 \\
\hline
\end{tabular}

the PBEPBE $/ 6-31+\mathrm{g}(\mathrm{d}, \mathrm{p})$ computation in the excited state shifted $\lambda_{\text {max }}$ by $+8 \mathrm{~nm}$.

The experimental $\lambda_{\max }$ value was simulated best by the use of PBEPBE $/ 6-31 \mathrm{G}+(\mathrm{d}, \mathrm{p})$ for both the ground state equilibrium geometry and the excited state calculations. However, PBEPBE/6-31G(d,p) is a more suitable method for the excited state calculations due to its having lesser computational time constraints .

\subsection{Solvent effect}

The solvent effect on the $\lambda_{\max }$ value was evaluated using the CPCM and IEF-PCM methods with various solvents having dielectric constant ranging from 9 to 78 . The computed and experimental $\lambda_{\max }$ values are displayed in Table 5 . This data shows that addition of solvent in the excited state shifts the wavelength by $15-20 \mathrm{~nm}$. On the other hand, the ground state geometry optimization in solvent shifts the wavelength by only $1-2 \mathrm{~nm}$. The results of the CPCM and IEF-PCM methods were very similar, having differences of only 2-3 nm. Any of these methods can be used for the calculations in solvent for this molecule. Table 5 shows that the $\lambda_{\max }$ values are almost independent of the polarity of the solvent, which is consistent with the experiments. The calculations are in fairly good agreement with experiment, with the computed wavelengths differing from the experimental value by approximately $10 \mathrm{~nm}$.

The transition nature with their coefficients and the oscillator strengths of TTBC in different solvents for the first excited states are also given in Table 5 . The $S_{1}$ excitation involves a dominant HOMO $\rightarrow$ LUMO with a coefficient of 0.6 for all solvents. All these transitions are intense with the oscillator strength values around 2. On the other hand, $\mathrm{S}_{2}$ transitions observed around $400 \mathrm{~nm}$ have dominant HOMO $\rightarrow$ LUMO +1 character with almost zero oscillator strength. The six frontier molecular orbitals of TTBC are given in Fig. 2. The electron densities are evenly distributed within the molecular skeleton of the HOMO. There is no electron density on the ethyl groups which are bonded to nitrogen atoms. The nitrogens have a nonbonding character in both the HOMO and LUMO.

Table 5

Experimental and calculated absorption $\lambda_{\max }$ (in $\mathrm{nm}$ ) and their transition natures, oscillator strengths of TTBC in different solvents.

\begin{tabular}{|c|c|c|c|c|}
\hline \multirow[t]{2}{*}{ Solvents } & \multicolumn{3}{|l|}{$\underline{\mathrm{S}_{1}}$} & \multirow{2}{*}{$\begin{array}{l}\text { Experimental [18] } \\
\lambda_{\text {abs }}\end{array}$} \\
\hline & $\lambda_{\mathrm{abs}}$ & $f$ & Transition character & \\
\hline Dichloromethane & 509 & 1.9694 & $\begin{array}{l}\mathrm{H} \rightarrow \mathrm{L}(0.6) \\
\mathrm{H} \rightarrow \mathrm{L}+2(0.1)\end{array}$ & - \\
\hline Acetone & 506 & 1.9428 & $\begin{array}{l}\mathrm{H} \rightarrow \mathrm{L}(0.6) \\
\mathrm{H} \rightarrow \mathrm{L}+2(0.1)\end{array}$ & - \\
\hline Ethanol & 506 & 1.9428 & $\begin{array}{l}H \rightarrow L(0.6) \\
H \rightarrow L+2(0.1)\end{array}$ & 516 \\
\hline Methanol & 504 & 1.9275 & $\begin{array}{l}\mathrm{H} \rightarrow \mathrm{L}(0.6) \\
\mathrm{H} \rightarrow \mathrm{L}+2(0.1)\end{array}$ & 514 \\
\hline Acetonitrile & 505 & 1.9361 & $\begin{array}{l}H \rightarrow L(0.6) \\
H \rightarrow L+2(0.1)\end{array}$ & 515 \\
\hline DMSO & 512 & 1.9833 & $\begin{array}{l}\mathrm{H} \rightarrow \mathrm{L}(0.6) \\
\mathrm{H} \rightarrow \mathrm{L}+2(0.1)\end{array}$ & 522 \\
\hline Water & 506 & 1.9265 & $\begin{array}{l}\mathrm{H} \rightarrow \mathrm{L}(0.6) \\
\mathrm{H} \rightarrow \mathrm{L}+2(0.1)\end{array}$ & - \\
\hline Gas & 486 & 1.7042 & $\begin{array}{l}\mathrm{H} \rightarrow \mathrm{L}(0.6) \\
\mathrm{H}-4 \rightarrow \mathrm{L}(0.1)\end{array}$ & $\begin{array}{l}- \\
-\end{array}$ \\
\hline
\end{tabular}

TDDFT/CPCM have been used with the PBEPBE $/ 631+G^{* *}$ level of theory on the DFT/ PBEPBE $/ 631+G^{* *}$ geometry. 


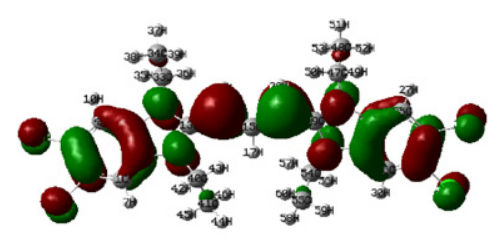

$\mathrm{HOMO}$

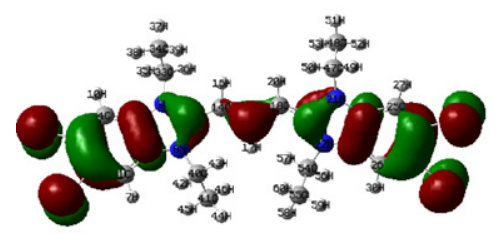

HOMO-1

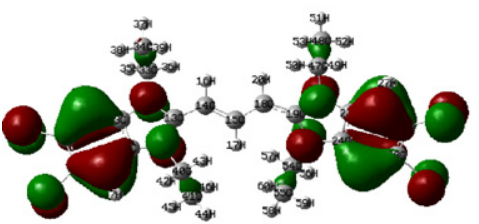

HOMO-2

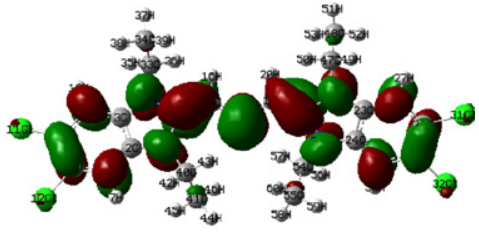

LUMO

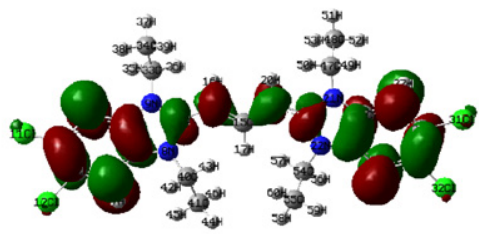

LUMO+1

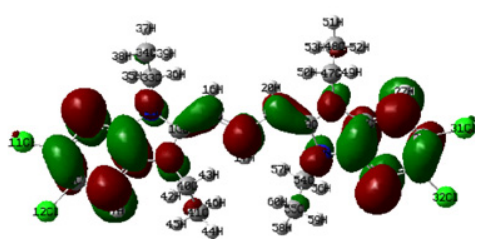

$\mathrm{LUMO}+2$

Fig. 2. Six frontier molecular orbitals of TTBC.

The electron density on the polymethine chain increases while the electron densities of chlorines decrease for the HOMO $\rightarrow$ LUMO transition. In contrast, the electrons moved from the nitrogen, chlorine atoms, and polymethine chain to the benzene rings for the $\mathrm{HOMO} \rightarrow \mathrm{LUMO}+1$. In HOMO- $\rightarrow$ LUMO and HOMO- $\rightarrow$ LUMO transitions, the electron migration from chlorines and benzimidazole rings to the polymethine chain was observed. HOMO-2 has no electron density on the polymethine chain and it is degenerate with the HOMO-1 state.

\subsection{Potential energy surface (PES) and fluorescence calculations}

To explore the rotational behavior of the molecule and to understand the fluorescence behavior of the dye, the ground state $\left(S_{0}\right)$, the excited singlet state $\left(S_{1}, S_{2}, S_{3}\right)$, and triplet state $\left(T_{1}, T_{2}, T_{3}\right)$ potential energy surface sections of TTBC were obtained as a function of torsional angles as shown in Fig. 3.

The molecule has been rotated around the $\mathrm{C} 13-\mathrm{C} 14\left(\theta_{1}\right)$ or $\mathrm{C} 14-\mathrm{C} 15\left(\theta_{2}\right)$ bond in increments of $15^{\circ}$ in the ground state equilibrium structure. The potential energy surface (PES) sections have been obtained by performing geometry optimizations using a frozen dihedral angle at each rotational point. All the energies have been given relative to the ground state minimum structure.

The excited states potential energy surface sections were explored by computing vertical excitation energies using the TDDFT//PBEPBE/6-31G(d,p) level of theory. The diffusion effects were ignored in the PES calculations because of computational cost.

Fig. 4 displays the PES section of TTBC as a function of $\theta_{1}$ for the singlet ground state, singlet excited states and triplet excited states. The gas phase results are shown with dashed lines, the solid lines are used for the results where methanol was used as solvent. The general trend of the all singlet surfaces are very similar in both methanol and gas phases. Solvent did not affect the $S_{0}$ state, but has small effects on the $S_{1}, S_{2}$ and $S_{3}$ states. Solvent stabilized $S_{2}$ and $S_{3}$ but destabilized $S_{1}$ when $\theta_{1}<90^{\circ}$. At $\theta_{1}=90^{\circ}$ solvent increased the energy of $S_{1}$ as shown in the inset of Fig. 4 and the higher states became degenerate.

The potential surfaces of both $S_{0}$ and $S_{1}$ are very shallow around the ground state minimum $\left(-50<\theta_{1}<50\right)$. Another minimum with a barrier of $0.09 \mathrm{eV}, 2.1 \mathrm{kcal} / \mathrm{mol}(0.11 \mathrm{eV}, 2.5 \mathrm{kcal} / \mathrm{mol}$ in

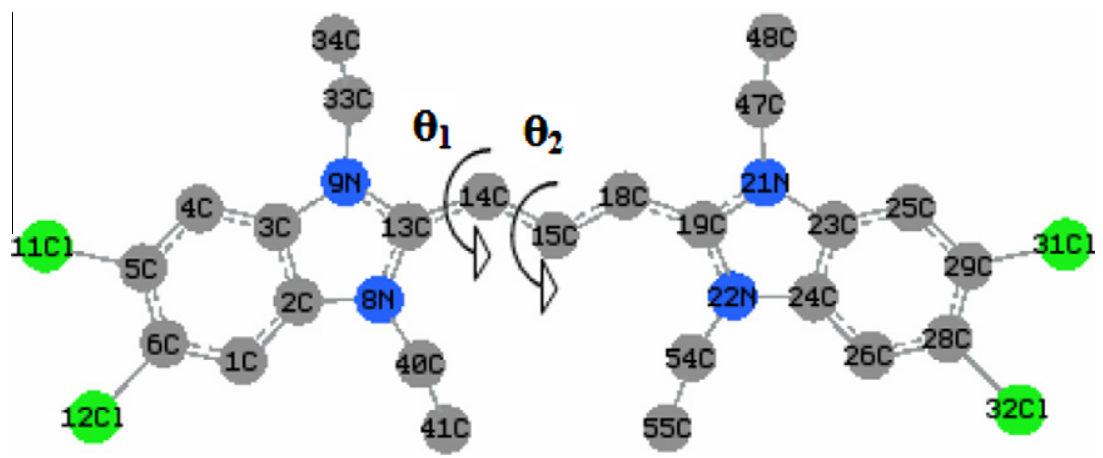

Fig. 3. Reaction coordinates $\left(\theta_{1}=\mathrm{N} 8-\mathrm{C} 13-\mathrm{C} 14-\mathrm{C} 15, \theta_{2}=\mathrm{C} 13-\mathrm{C} 14-\mathrm{C} 15-\mathrm{C} 18\right)$ used in PES calculations. 


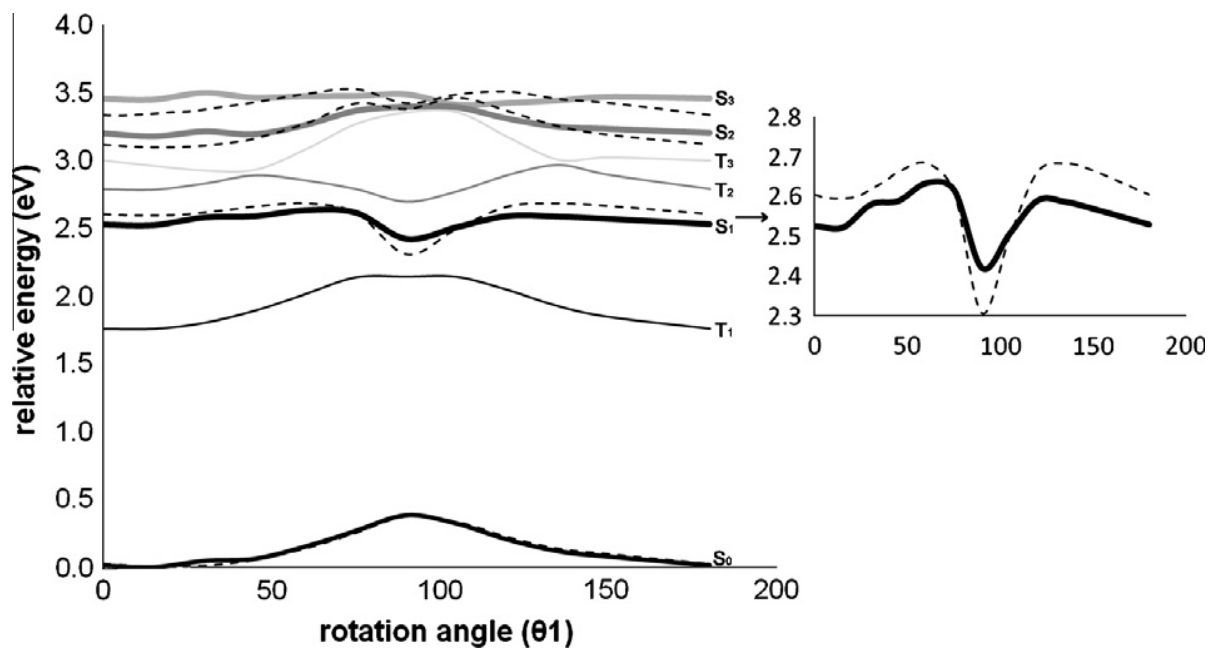

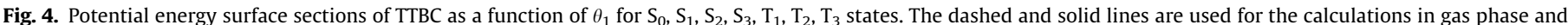
methanol respectively.

methanol) was detected in the first excited state surface at $\theta_{1}=90^{\circ}$ and there is a maximum at the same point in the ground state (inset, Fig. 4). The barrier is located at $\theta_{1}=75^{\circ}$. In methanol the excitation energies from the ground state minimum structure and perpendicular structures are $2.52 \mathrm{eV}(491 \mathrm{~nm})$ and $2.04 \mathrm{eV}$ $(609 \mathrm{~nm})$, respectively.

The triplet excited state surfaces in methanol are also given in Fig. 4. $\mathrm{T}_{2}$ and $\mathrm{S}_{1}$ energies differ by about $0.26 \mathrm{eV}(6 \mathrm{kcal} / \mathrm{mol}$; $25 \mathrm{~kJ} / \mathrm{mol}$ ) around the equilibrium $S_{0} . T_{2}$ also has a minimum, $T_{1}$ has a maximum, and $S_{2}, S_{3}$ and $T_{3}$ are all degenerate for the twisted structure. The energies of $S_{1}$ and $T_{1}$ are separated by $0.28 \mathrm{eV}$ ( $6.5 \mathrm{kcal} / \mathrm{mol} ; 27 \mathrm{~kJ} / \mathrm{mol}$ ) for the orthogonal structure. $\mathrm{T}_{2}$ and $\mathrm{T}_{3}$ become degenerate at rotational angles of $45^{\circ}$ and $135^{\circ}$ where they have a maximum and minimum, respectively. The nature of $\mathrm{T}_{1}$ and $\mathrm{S}_{1}$ states is HOMO to LUMO transition at all rotational angles, while the nature of $S_{2}$ is composed of HOMO to LUMO + 1(with coefficient around 0.6) and HOMO-1 to LUMO (with coefficient around $0.2-0.4$ ) except $\theta_{1}=90^{\circ}$ and $105^{\circ}$ where the nature becomes HOMO to LUMO +2 . On the other hand, $\mathrm{T}_{2}$ has HOMO-1 to LUMO transition when $0^{\circ} \leqslant \theta_{1} \leqslant 45^{\circ}$ and $150^{\circ} \leqslant \theta_{1} \leqslant 180^{\circ}$ and HOMO to LUMO + 1 when $45^{\circ} \leqslant \theta_{1} \leqslant 135^{\circ}$. The character of $S_{3}$ is a mixture of of HOMO to LUMO + 2 (with coefficient around 0.50.6 ) and HOMO-3 to LUMO (with coefficient around 0.2-0.4) except $\theta_{1}=90^{\circ}$ and $105^{\circ}$ where the character turns out to be HOMO to LUMO +1 . For $\mathrm{T}_{3}$, HOMO to LUMO +1 is the origin of the transition when $0^{\circ} \leqslant \theta_{1} \leqslant 30^{\circ}$ and $150^{\circ} \leqslant \theta_{1} \leqslant 180^{\circ}$, HOMO- 1 to LUMO when $45^{\circ} \leqslant \theta_{1} \leqslant 75^{\circ}$ and $120^{\circ}, 135^{\circ}$, HOMO to LUMO + 2 at $90^{\circ}$ and HOMO-2 to LUMO at $105^{\circ}$. Although $\mathrm{T}_{2}$ and $\mathrm{S}_{1}$ states have similar shape in Fig. 4, they do not have the same nature.

These results suggest that, there might be two emission sources. In other words, the molecule might have a dual emission. An experimental fluorescence maximum appears at $528 \mathrm{~nm}$ with a shoulder located at $560 \mathrm{~nm}$ in methanol; however, the bathochromic shift with solvent polarity was not observed. The dual emission was suggested experimentally only in glycerol where a double exponential decay was detected [18]. However there is no experimental evidence of a charge transfer state for that dye. The close energies of $T_{2}$ and $S_{1}$ may cause the delay of the fluorescence signal from the second emission source. PES sections were also obtained as a function of $\theta_{2}$. However, the $S_{1}$ surface is almost flat, and is not affected by this rotation.

Fig. 5 includes the energy profiles of six frontier orbitals (three virtual and three occupied) as a function of $\theta_{1}$ in the gas phase and methanol. The degeneracy in HOMO-2 and HOMO-1 are not disturbed by the rotation. The highest two LUMO's are close to each other. The solvent causes to increase all the orbital energies.

The excited state geometry optimizations were performed to evaluate the fluorescence behavior of the TTBC molecule. The

\section{(a)}

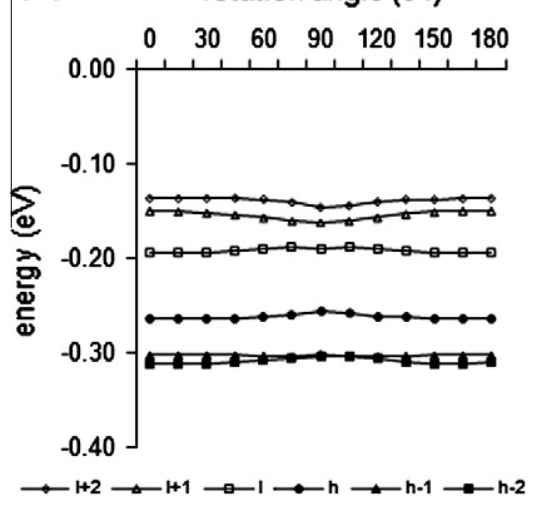

(b) rotation angle $(\theta 1)$

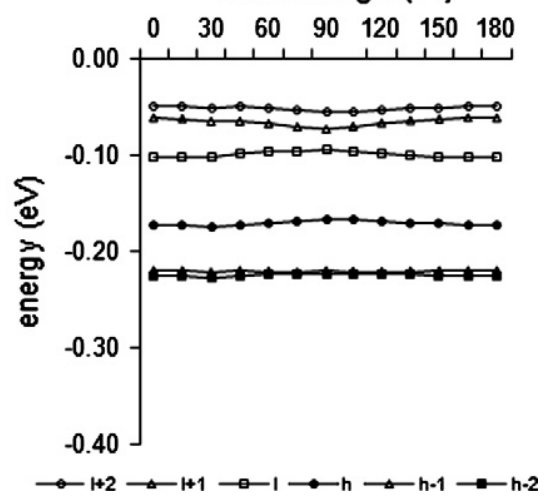

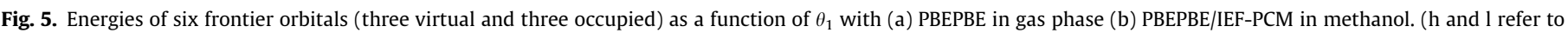
HOMO and LUMO respectively). 
ground state equilibrium and some twisted structures were chosen as the initial geometries for the geometry optimization at the TDDFT/PBEPBE/6-31G(d,p) level of theory. The results provided two different structures. Starting with the ground state geometry, a structure very similar to the ground state was calculated. For this minimum configuration, the dihedral angle $\left(\theta_{1}\right)$ in the excited state is lowered with respect to the ground state by $5^{\circ}$ and $9^{\circ}$ in the gas phase and methanol, respectively. This shows that the TTBC molecule tends to become planar upon excitation.

The remaining initial structures with the twisted geometries $\left(\theta_{1}=75^{\circ}, 90^{\circ}, 105^{\circ}\right)$ converged into another twisted configuration having $\theta_{1}=117^{\circ}\left(115^{\circ}\right.$ in methanol). The energy of this structure in the gas phase is $0.92 \mathrm{eV}(21.2 \mathrm{kcal} / \mathrm{mol} ; 88.8 \mathrm{~kJ} / \mathrm{mol})$ lower that that of the more planar structure. Likewise, when methanol is present, the energy difference in the structure becomes $0.25 \mathrm{eV}$ (5.8 kcal $/ \mathrm{mol} ; 24.1 \mathrm{~kJ} / \mathrm{mol}$ ) lower than that of the more planar one.

Some selected geometric parameters in the $\mathrm{S}_{0}$ equilibrium structure and corresponding relaxed structure in $S_{1}$ are given in Table 6 . When equilibrium ground state structure is compared to the relaxed $S_{1}$ structure, some of the bonds are elongated while others are shortened. Although there are no dramatic changes in the relaxed structure, there are some small changes in the imidazole ring and polymethine chain for both gas phase and methanol cases. These changes are observed as a decrease $(0.01 \AA)$ in $\mathrm{N} 8-\mathrm{C} 2$, N9-C3, C13-C14 and as an increase $(0.01-0.017 \AA)$ in $\mathrm{C} 2-\mathrm{C} 3$, $\mathrm{C} 14-\mathrm{C} 15, \mathrm{~N} 8-\mathrm{C} 13, \mathrm{~N} 9-\mathrm{C} 13$, bond lengths. The bond angles that involve nitrogens and polymethine carbons are also effected slightly $\left( \pm 1.2^{\circ}\right.$ in methanol). As a summary, upon excitation, the most effected moieties of the dye are imidazole and polymethine chain.

When both minimum configurations at the $S_{1}$ surface are compared in the gas phase, $\mathrm{C} 13-\mathrm{C} 14$ and $\mathrm{C} 14-\mathrm{C} 15$ bonds become longer by about $0.1 \mathrm{~A}$ at the twisted configuration. The N8-C13 and $\mathrm{N} 9-\mathrm{C} 13$ bonds are also elongated by $0.02 \AA$. The $\mathrm{C} 15-\mathrm{C} 14-\mathrm{C} 13$ and $\mathrm{C}(14)-\mathrm{C}(13)-\mathrm{N}(8)$ angles are increased by twisting approximately $8-14^{\circ}$. Similar behaviors are found in the methanol phase.
The Mulliken charge analysis was evaluated to compare ground and excited state charge distributions. For the first minimum, similar to geometry comparison, although there are no considerable changes of charges upon excitation, the charges of the polymethine carbons are altered slightly as an alternating decrease and increase $(-0.02 ;+0.035$ au in gas phase, $-0.04 ;+0.035$, in methanol). The charges on the chlorines are also increased by 0.02 units in the gas phase. These results explain the solvent independency of absorption and fluorescence spectrum on TTBC.

For the twisted configuration of the TTBC molecule, the charges in the excited state were compared with the corresponding ground state charges. The largest change was observed on the chlorines and five atoms of the benzimidazole ring on one side of the molecule and on two of the polymethine carbons. The charges of $\mathrm{C} 1, \mathrm{C} 4$, $\mathrm{N} 8, \mathrm{~N} 9, \mathrm{C} 13$ and $\mathrm{Cl}-11, \mathrm{Cl}-12$ decreased whereas the charges on $\mathrm{C} 14, \mathrm{C} 18, \mathrm{Cl}-31, \mathrm{Cl}-32$ increased. It should be noted that all the decreases are observed on one side; however, all increases are observed on the opposite side of the molecule. When all increases and decreases are summed, the net result is \pm 0.5 au. Similar results were obtained from the same calculations in methanol. The emission from this state would have a charge transfer character.

The fluorescence wavelengths in different solvents are given in Table 7. The fluorescence wavelength shifted by $66-68 \mathrm{~nm}$ via the solvent effect while the polarity of the solvent did not affect the fluorescence emission line (as similar to the experimental observation). The Stokes shift is about $30 \mathrm{~nm}$ in methanol compared to an experimental value of $14 \mathrm{~nm}$.

Table 7

The calculated and experimental fluorescence wavelengths (in $\mathrm{nm}$ ) in different solvents.

\begin{tabular}{lll}
\hline Solvents & IEFPCM $\lambda_{\text {fluo }}$ & Experiment $\lambda_{\text {fluo }}$ \\
\hline Gas phase & 497 & - \\
Ethanol & 563 & 530 \\
Methanol & 564 & 528 \\
Acetonitrile & 564 & 529 \\
DMSO & 565 & 536 \\
\hline
\end{tabular}

Table 6

Some selected structural parameters of TTBC in the $S_{0}$ and $S_{1}$ states.

\begin{tabular}{|c|c|c|c|c|c|c|}
\hline \multirow[t]{2}{*}{ Atoms } & \multicolumn{3}{|l|}{ Gas } & \multicolumn{3}{|l|}{ Methanol } \\
\hline & $\mathrm{S}_{0}$ & $\mathrm{~S}_{1}$ & $\mathrm{~S}_{1}^{\text {(twisted) }}$ & $\mathrm{S}_{0}$ & $\mathrm{~S}_{1}$ & $\mathrm{~S}_{1}^{\text {(twisted) }}$ \\
\hline$C(1)-C(2)$ & 1.396 & 1.396 & 1.397 & 1.396 & 1.401 & 1.400 \\
\hline$C(2)-C(3)$ & 1.410 & 1.421 & 1.420 & 1.412 & 1.421 & 1.423 \\
\hline$C(3)-C(4)$ & 1.396 & 1.398 & 1.398 & 1.397 & 1.403 & 1.401 \\
\hline$C(4)-C(5)$ & 1.405 & 1.409 & 1.413 & 1.404 & 1.407 & 1.417 \\
\hline$C(5)-C(6)$ & 1.421 & 1.423 & 1.409 & 1.416 & 1.419 & 1.402 \\
\hline$C(6)-C(1)$ & 1.405 & 1.410 & 1.414 & 1.405 & 1.408 & 1.417 \\
\hline $\mathrm{C}(5)-\mathrm{Cl}(11)$ & 1.736 & 1.732 & 1.742 & 1.747 & 1.745 & 1.757 \\
\hline $\mathrm{C}(6)-\mathrm{Cl}(12)$ & 1.736 & 1.732 & 1.742 & 1.747 & 1.745 & 1.757 \\
\hline $\mathrm{C}(2)-\mathrm{N}(8)$ & 1.396 & 1.387 & 1.385 & 1.392 & 1.381 & 1.381 \\
\hline $\mathrm{N}(8)-\mathrm{C}(13)$ & 1.384 & 1.400 & 1.421 & 1.385 & 1.398 & 1.413 \\
\hline $\mathrm{N}(9)-\mathrm{C}(13)$ & 1.386 & 1.403 & 1.418 & 1.388 & 1.402 & 1.411 \\
\hline $\mathrm{N}(9)-\mathrm{C}(3)$ & 1.392 & 1.382 & 1.382 & 1.388 & 1.376 & 1.380 \\
\hline$N(9)-C(33)$ & 1.467 & 1.464 & 1.461 & 1.468 & 1.467 & 1.463 \\
\hline $\mathrm{N}(8)-\mathrm{C}(40)$ & 1.465 & 1.465 & 1.462 & 1.468 & 1.468 & 1.463 \\
\hline$C(13)-C(14)$ & 1.412 & 1.401 & 1.505 & 1.410 & 1.405 & 1.489 \\
\hline$C(14)-C(15)$ & 1.402 & 1.417 & 1.350 & 1.402 & 1.414 & 1.366 \\
\hline $\mathrm{N}(8)-\mathrm{C}(2)-\mathrm{C}(1)$ & 131.4 & 131.1 & 131.3 & 131.2 & 131.1 & 131.4 \\
\hline$N(9)-C(33)-C(34)$ & 113.0 & 113.2 & 113.3 & 112.9 & 112.9 & 105.1 \\
\hline $\mathrm{N}(9)-\mathrm{C}(13)-\mathrm{N}(8)$ & 106.9 & 106.2 & 104.7 & 107.0 & 106.2 & 105.1 \\
\hline$N(9)-C(3)-C(4)$ & 131.7 & 131.5 & 131.3 & 131.6 & 131.4 & 131.3 \\
\hline $\mathrm{Cl}(11)-\mathrm{C}(5)-\mathrm{C}(6)$ & 120.8 & 120.7 & 121.1 & 120.8 & 120.7 & 121.1 \\
\hline $\mathrm{Cl}(11)-\mathrm{C}(5)-\mathrm{C}(4)$ & 118.2 & 118.2 & 117.8 & 118.0 & 117.9 & 117.4 \\
\hline$C(15)-C(18)-C(19)$ & 129.9 & 130.5 & 130.4 & 129.9 & 131.2 & 130.0 \\
\hline$C(15)-C(14)-C(13)$ & 129.9 & 130.5 & 122.4 & 129.9 & 131.2 & 122.1 \\
\hline $\mathrm{C}(14)-\mathrm{C}(13)-\mathrm{N}(8)$ & 130.3 & 130.2 & 116.3 & 130.5 & 130.7 & 119.7 \\
\hline $\mathrm{N}(8)-\mathrm{C}(13)-\mathrm{C}(14)-\mathrm{C}(15)$ & 19.1 & 13.8 & 117.3 & 17.5 & 8.8 & 114.5 \\
\hline
\end{tabular}


The experimental [18] and simulated absorption and fluorescence spectra in methanol are compared in Fig. 6. The computed absorption spectrum was obtained by the TDDFT/PBEPBE/631+G $(d, p) / I E F-P C M$ level of theory while the diffusion function correction was not used for the simulation of the fluorescence spectrum.

Computational spectra were normalized at the wavelength maximum of TTBC. The computed absorption spectrum shows good agreement with its experimental $\lambda_{\max }$ value. The fluorescence $\lambda_{\max }$ was a bit different from the experimental value by about $30 \mathrm{~nm}$ which is also reasonable with the level of theory used in the calculation since diffusion functions were not used. The shoulders which appear in the experimental spectra at $480 \mathrm{~nm}$ (absorption) and $560 \mathrm{~nm}$ (emission) did not show up in the simulated spectra.

The six molecular orbitals of TTBC for the twisted optimized structure of the $S_{1}$ state in gas phase are displayed in Fig. 7. The electrons are localized on one side of the molecule in occupied orbitals whereas they are placed on the opposite side of the molecule in unoccupied orbitals. There is a clear migration of electrons from one side to the other side of the TTBC molecule. HOMO and HOMO-2 have electron densities on the polymethine chain but HOMO-1 does not. All the transitions for this configuration have almost zero oscillator strength. The emission from the twisted $S_{1}$ state is observed at $5101 \mathrm{~nm}$ and $1291 \mathrm{~nm}$ in the gas phase and methanol, respectively, and is mainly HOMO to LUMO character (0.7). The next transition is located at $801 \mathrm{~nm}$ and $478 \mathrm{~nm}$ in the gas phase and methanol, respectively, being HOMO-1 to LUMO in nature (0.7).

There is a noticeable dipole change in the $S_{1}$ state for the twisted geometry as shown in Fig. 8. This is an indication of a charge transfer. The HOMO-LUMO pictures also allow us to propose a charge transfer existing from one side of the molecule to the other side (Fig. 7) for the twisted geometry. The dipoles in $S_{1}$ and $S_{0}$ are similar in magnitude to the ground state structure, which is also supported by the experiment. On the other hand, the dipoles of the optimized twisted structure in the excited state are 15D and 19D in the gas phase and methanol, respectively, while the corresponding ground state dipoles are about 9D.

Recently, measurements and simulations of the polarized absorption spectra of TTBC aggregates dispersed in polymer thin films have been performed to determine their structure-spectroscopy function relationship [65]. It has been observed that, two aggregate bands ( $\mathrm{H}$ and $\mathrm{J}$ ) have opposite polarization dependence. The H-band has a maximum intensity when the electric field vector and the macroscopic alignment axis are almost parallel to each other and the H-band has a minimum intensity when these two vectors are almost perpendicular to each other. However, the J band's behavior was found to be just the opposite of this. The
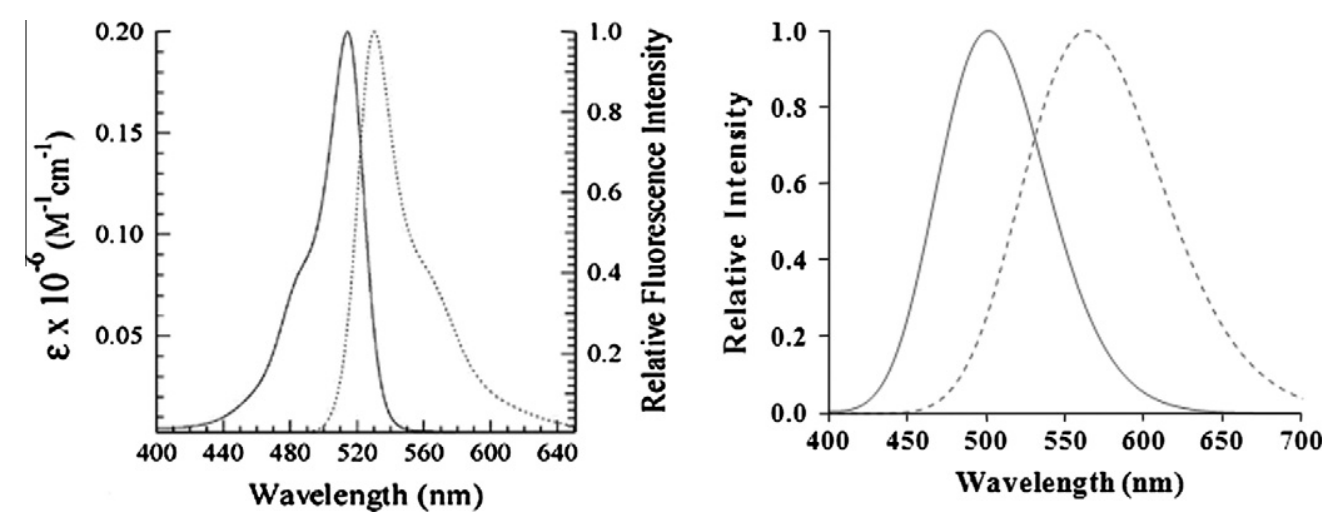

Fig. 6. Absorption and fluorescence spectra of TTBC: (a) experimental (b) computed.

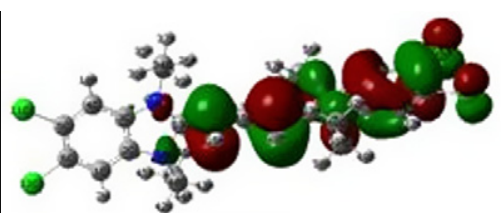

HOMO

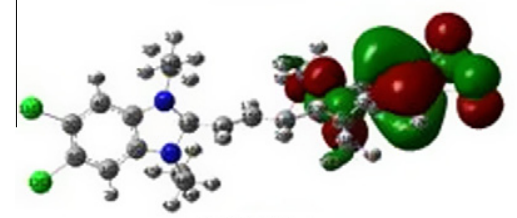

HOMO-1

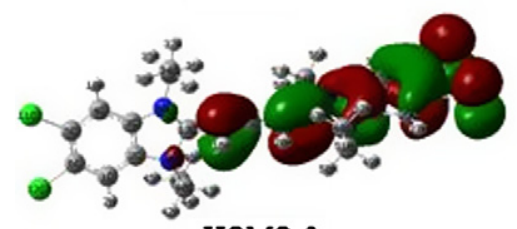

HOMO-2

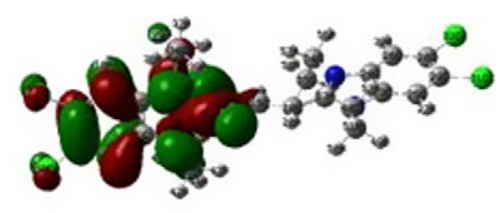

LUMO

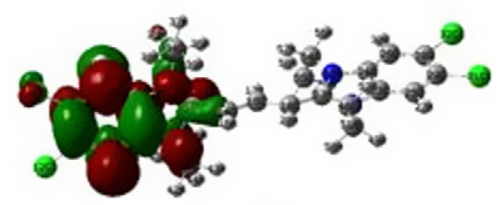

$\mathrm{LUMO}+1$

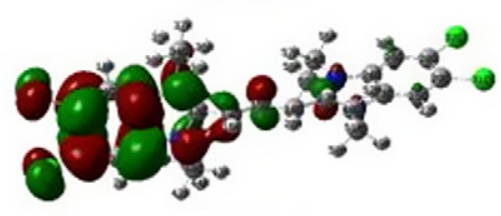

LUMO +2

Fig. 7. The six frontier molecular orbitals of TTBC for the $S_{1}$ state twisted configuration. 


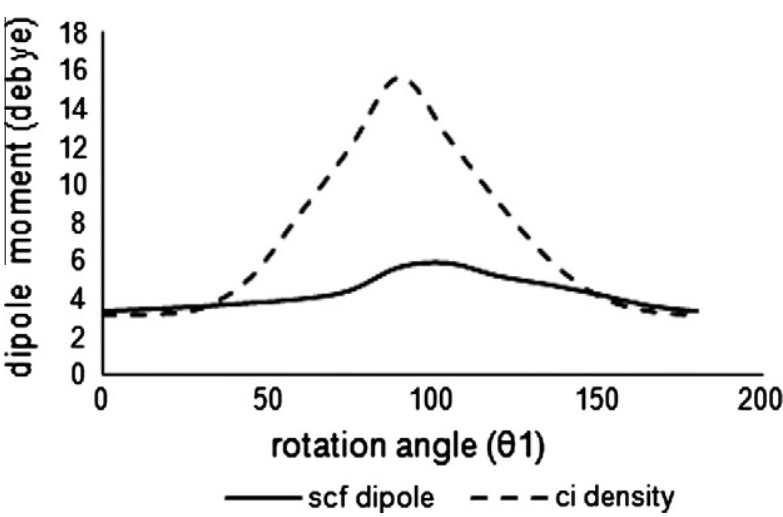

Fig. 8. Ground and excited state dipoles of TTBC.

unpublished fluorescence anisotropy measurements performed by Özçelik reveal that when the angle between the polarizer and the fluorescence state is perpendicular, maximum fluorescence intensity is obtained at $590 \mathrm{~nm}$ for the TTBC aggregates (J-aggregate) which is important to monitor cell apoptosis. The second minimum in the $S_{1}$ state might be important for understanding and improving all these aggregation behaviors of the TTBC dye.

\section{Conclusions}

In this work, the ground state and excited state behavior of TTBC dye in the gas phase and solvents was examined by using quantum chemical methods. The PBEPBE functional with 6$31 \mathrm{G}(\mathrm{d}, \mathrm{p})$ can be used reliably for both ground state and excited state calculations for this dye. The TDDFT method gave results consistent with experiment in the calculation of the absorption wavelength. It was shown that this was also true for calculations of the fluorescence wavelength for this dye.

There are two distinct minima on the $\mathrm{S}_{1}$ surface located around the ground state equilibrium geometry and twisted geometry, the latter of which is the energetically favorable one. The twisted geometry also has a charge transfer character and might be connected to the ground state equilibrium geometry by a barrier. This barrier is small according to the $S_{1}$ PES section obtained by vertical excitations from the relaxed ground state optimized geometries. However it is difficult to say whether these two minima are connected with a low or high barrier; further work should be done to find the pathways between these two minima. There is no experimental evidence about the twisted state. This might be explained by either a high barrier or an intersystem crossing to the second triplet excited state which is close in energy to $S_{1}$, possibly delaying the fluorescence signal emitted from this state. There might also be an internal conversion, radiationless transition from the $S_{1}$ to $S_{0}$ states since their separation is about $5 \mathrm{kcal} / \mathrm{mol}$ and $20 \mathrm{kcal} / \mathrm{mol}$ in the gas phase and in methanol solvent, respectively.

The ground and excited state dipoles are almost same near the equilibrium ground state geometry. This can be explained by there being no drastic change of charges and geometrical parameters. The TTBC molecule becomes more planar upon excitation around the ground state. The fluorescence and absorption $\lambda_{\max }$ values are independent of the polarity of the solvents. All these observations are supported by experimental results. This reveals that, there is almost no competition between the two minima in the $S_{1}$ state and the fluorescence emission is predominantly coming from the more planar configuration.

This work provides useful information for the new molecular design and the modification of the electronic and optical properties of TTBC-based versatile cyanine dyes. The twisted charge transfer state might be made active by the modification of the TTBC molecule with different functional groups.

\section{Acknowledgment}

We would like to thank to Serdar Özçelik of IYTE, for inspirations to this study and help of the experimental part. We also like to thank to Ritchie Eanes for the proof reading of the manuscript.

\section{References}

[1] IUPAC. Compendium of Chemical Terminology, second ed. Blackwell Scientific Publications, Oxford, 1997. p. 1329. <http://goldbook.iupac.org/C01487.html> (accessed 26.03.09).

[2] A. Mishra, R.K. Behera, P.K. Behera, B.K. Mishra, G.B. Behera, Chem. Rev. 100 (2000) 1973

[3] J.R. Lakowicz, Topics in Fluorescence Spectroscopy, Probe Design and Chemical Sensing, vol. 4, Plenum Press, New York and London, 1994.

[4] J.F. Hamilton, in: T.H. James (Ed.), The Theory of the Photographic Process, 4th ed., Macmillan, New York, 1977, p. 235.

[5] F.P. Schäfer, Dye Lasers, 3rd ed., Springer-Verlag, New York, 1990.

[6] T.G. Pavlopoulos, Laser dye structure and spectroscopic properties, in: H.S. Freeman, A. Peters (Eds.), Colorants for Non-Textile Applications, Elsevier, Amsterdam, 2000, p. 274

[7] M. Kawakami, K. Koya, T. Ukai, N. Tatsuta, A. Ikegawa, K. Ogawa, T. Shishido, L.B. Chen, J. Med. Chem. 41 (1998) 130.

[8] M. Furuki, O. Wada, L.S. Pu, L.Y. Sato, H. Kawashima, T. Tani, J. Phys. Chem. B 103 (1999) 7607.

[9] P. Chen, S. Sun, Y. Hu, Z. Qian, D. Zheng, Dyes Pigm. 41 (1999) 227.

[10] R.P. Haugland, Handbook of Fluorescent Probes and Research Chemicals, fifth ed., Molecular Probes, Eugene, Oregon, 1992.

[11] A.S. Waggoner, Cyanine Dyes as Labeling Reagents for Detection of Biological and Other Materials by Luminescence Methods. US Patent 6956,032 Bl, October, 18, 2005.

[12] E.E. Jelley, Nature 138 (1936) 1009.

[13] G. Scheibe, Angew. Chem. 50 (1937) 51

[14] M. Reers, T.W. Smith, L.B. Chen, Biochemistry 30 (1991) 4480

[15] S. Smiley, M. Reers, H.C. Mottola, M. Lin, A. Chen, T.W. Smith, G.D. Steele Jr., L.B. Chen, Proc. Natl. Acad. Sci. USA 88 (1991) 3671.

[16] A. Cossarizza, C.M. Baccarani, G. Kalashnikova, C. Franchesci, Biochim. Biophys. Res. Commun. 197 (1993) 40.

[17] E. Simeonova, M. Garstka, J. Kozioł-Lipińska, A. Mostowska, Protoplasma 223 (2004) 143.

[18] S. Özçelik, J. Lumin. 96 (2002) 141.

[19] E. Runge, E.K.U. Gross, Phys. Rev. Lett. 52 (1984) 997.

[20] M.A.L. Marques, E.K.U. Gross, Annu. Rev. Phys. Chem. 55 (2004) 427.

[21] M.E. Casida, in: Recent Advances in Density Functional Methods, Part I, World Scientific, Singapore, 1995.

[22] L.Y. Wang, Q.W. Chen, G.H. Zhai, Z.Y. Wen, Z.X. Zhang, Dyes Pigm. 72 (2007) 357.

[23] P.H. de-Mello, B. Mennucci, J. Tomasi, A.B.F. da Silva, Theor. Chem. Acc. 118 (2007) 305.

[24] M. Sun, B. Niu, J. Zhang, Theor. Chem. Acc. 119 (2008) 489.

[25] Y.P. Lu, M. Ehara, Theor. Chem. Acc. 124 (2009) 395

[26] E. Benassi, F. Spagnolo, Theor. Chem. Acc. 124 (2009) 235

[27] Z. Lui, Dyes Pigm. 85 (2010) 109.

[28] P. Song, F. Ma, J. Phys. Chem. A 114 (2010) 2230.

[29] A. Bamgbelu, J. Wang, J. Leszczynski, J. Phys. Chem. A 114 (2010) 3551.

[30] D. Casanova, F.P. Rotzinger, M. Grätzel, J. Chem. Theory Comput. 6 (2010) 1219.

[31] D. Jacquemin, J. Preat, V. Wathelet, E.A. Perpéte, J. Mol. Struct. (Theochem.) 731 (2005) 67.

[32] D. Jacquemin, J. Preat, E.A. Perpète, Chem. Phys. Lett. 410 (2005) 254.

[33] D. Jacquemin, J. Preat, V. Wathelet, E.A. Perpète, Chem. Phys. 328 (2006) 324

[34] D. Jacquemin, J. Preat, V. Wathelet, E.A. Perpète, J. Chem. Phys. 124 (2006) 074104.

[35] D. Jacquemin, J. Preat, V. Wathelet, M. Fontaine, E.A. Perpète, J. Am. Chem. Soc. 128 (2006) 2072

[36] D. Jacquemin, E.A. Perpète, G.E. Scuseria, I. Ciofini, C. Adamo, J. Chem. Theory Comput. 4 (2008) 123.

[37] D. Jacquemin, E.A. Perpéte, G. Scalmani, M.J. Frisch, X. Assfeld, I. Ciofini, C. Adamo, J. Chem. Phys. 125 (2006) 164324.

[38] D. Jacquemin, C. Peltier, I. Ciofini, Chem. Phys. Lett. 493 (2010) 67

[39] D. Jacquemin, E.A. Perpète, C. Adamo, Int. J. Quantum Chem. 110 (2010) 2121

[40] J. Preat, D. Jacquemin, E.A. Perpète, Int. J. Quantum Chem. 110 (2010) 2147.

[41] Z.L. Cai, K. Sendt, J.R. Reimers, J. Chem. Phys. 117 (2002) 5543.

[42] S. Grimme, M. Parac, Chem. Phys. Chem 3 (2003) 292.

[43] D.J. Tozer, N.C. Handy, J. Chem. Phys. 109 (1998) 10180.

[44] S. Hirata, C.G. Zhan, E. Aprá, T.L. Windus, D.A. Dixon, J. Phys. Chem. A 107 (2003) 10154.

[45] D.J. Tozer, N.C. Handy, Phys. Chem. Chem. Phys. 2 (2000) 2117.

[46] S. Hirata, M.H. Gordon, Chem. Phys. Lett. 302 (1999) 375.

[47] N.T. Maitraa, F. Zhang, R. Cave, K. Burke, J. Chem. Phys. 120 (2004) 5932.

[48] A. Dreuw, M.H. Gordon, J. Am. Chem. Soc. 126 (2004) 4007 
[49] D.J. Tozer, R.D. Amos, N.C. Handy, B.O. Roos, L.S. Andres, Mol. Phys. 97 (1999) 859.

[50] T. Stein, L. Kronik, R. Baer, J. Am. Chem. Soc. 131 (2009) 2818.

[51] M.J. Frisch, G.W. Trucks, H.B. Schlegel, G.E. Scuseria, M.A. Robb, J.R. Cheeseman, et al. Gaussian 09, revision A.02, Gaussian, Inc, Wallingford CT, 2009.

[52] J.P. Perdew, K. Burke, M. Ernzerhof, Phys. Rev. Lett. 77 (1996) 3865.

[53] M. Ernzerhof, G.E. Scuseria, J. Chem. Phys. 110 (1999) 5029.

[54] C. Adamo, V. Barone, J. Chem. Phys. 110 (1999) 6158.

[55] A.D. Becke, J. Chem. Phys. 98 (1993) 5648.

[56] T. Yanai, D. Tew, N. Handy, Chem. Phys. Lett. 393 (2004) 51.
[57] M.T. Cancès, B. Mennucci, J. Tomasi, J. Chem. Phys. 107 (1997) 3032.

[58] B. Mennucci, J. Tomasi, J. Chem. Phys. 106 (1997) 5151.

[59] B. Mennucci, E. Cancès, J. Tomasi, J. Phys. Chem. B 101 (1997) 10506.

[60] J. Tomasi, B. Mennucci, E. Cancès, J. Mol. Struct. (Theochem.) 464 (1999) 211

[61] V. Barone, M. Cossi, J. Phys. Chem. A 102 (1998) 1995.

[62] M. Cossi, N. Rega, G. Scalmani, V. Barone, J. Comp. Chem. 24 (2003) 669.

[63] D.L. Smith, H.R. Luss, Acta Crystall. Sec. B 28 (1972) 2793.

[64] S. Karaca, N. Elmacı, J. Mol. Struct. (Theochem.) 915 (2009) 149.

[65] D. Gülen, O. Atasoylu, S. Özçelik, Chem. Phys. 355 (2009) 73. 